\title{
PADÉ APPROXIMATIONS TO THE LOGARITHM III: ALTERNATIVE METHODS AND ADDITIONAL RESULTS
}

\author{
KATHY DRIVER, HELMUT PRODINGER, CARSTEN SCHNEIDER, AND J.A.C. WEIDEMAN
}

\begin{abstract}
We use C. Schneider's summation software Sigma and a method due to AndrewsNewton-Zeilberger to reprove results from [5] and [24] as well as to prove new related material.
\end{abstract}

\section{INTRODUCTION}

In [24], the problem of finding Padé approximations to the logarithm based at $x=1$ was studied. In the sequel [5] we derived a number of recurrences and combinatorial identities related to this problem. During the course of this study, alternative proofs were found and additional identities were derived with a different method (due to Andrews-Newton-Zeilberger). These additional identities are most likely new, beautiful, and challenging, and we therefore decided to collect this additional material in part III of the present series.

To give an idea of these identities we mention

$$
\sum_{k=0}^{2 m}(-1)^{k}\left(\begin{array}{c}
2 m \\
k
\end{array}\right)^{3}\left[3 H_{k}^{2}-3 H_{k} H_{2 m-k}+H_{k}^{(2)}\right]=0, \quad m \geq 0,
$$

which was proved in [5], but will be re-derived in Section 2 below with a different method. Three identities believed to be new are

$$
\begin{aligned}
\sum_{k=0}^{2 m}(-1)^{k}\left(\begin{array}{c}
2 m \\
k
\end{array}\right)^{3} H_{k} & =\frac{(-1)^{m}}{2} \frac{(3 m) !}{(m !)^{3}}\left[H_{m}+2 H_{2 m}-H_{3 m}\right], \\
\sum_{k=0}^{2 m}(-1)^{k}\left(\begin{array}{c}
2 m \\
k
\end{array}\right)^{3} H_{k}^{(2)} & =\frac{(-1)^{m}}{2} \frac{(3 m) !}{(m !)^{3}}\left[H_{m}^{(2)}+H_{2 m}^{(2)}\right]
\end{aligned}
$$

both of which will be proved in Section 2, and

$$
\sum_{k=0}^{m-j-1}(-1)^{k} \frac{1}{m-j-k}\left(\begin{array}{c}
2 k \\
k
\end{array}\right)\left(\begin{array}{c}
2 m+k \\
2 k
\end{array}\right)=\frac{(-1)^{m-j}(3 m-j) !}{(m-j) !^{2}(m+j) !}\left[H_{m+j}-2 H_{2 m}+H_{3 m-j}\right],
$$

for $|j| \leq m$, which will be proved in Section 4 .

Date: October 5, 2006.

1991 Mathematics Subject Classification. 41A21, 05A19, 33F10.

Key words and phrases. Padé approximation, combinatorial identities, computer algebra, creative telescoping. 


\section{The method of Andrews-Newton-Zeilberger}

G. Andrews discusses in $[2,3]$ a method to prove identities involving harmonic numbers. This was recently developed further by one of the authors and P. Paule into an algorithmic method [12]. A variation of this strategy will be employed here to prove identities (1)-(3) with purely hypergeometric methods.

We shall illustrate this so-called Andrews-Newton-Zeilberger method [12] by first proving the identity (2). The idea is based on the following crucial observation. Let $L$ be the operator that evaluates functions $f(x)$ at $x=0$, i.e., $L f(x):=f(0)$. Let $D$ be differentiation with respect to $x$, i.e., $D f(x):=f^{\prime}(x)$. It is an easy exercise to verify that for all integers $m$,

$$
L D\left(\begin{array}{c}
x+m \\
m
\end{array}\right)=H_{m}
$$

Now denote the left side of (2) by $G_{m}$, i.e.,

$$
G_{m}:=\sum_{k=0}^{2 m}(-1)^{k}\left(\begin{array}{c}
2 m \\
k
\end{array}\right)^{3} H_{k}
$$

Instead of computing a recurrence for this expression, we search for a recurrence of the hypergeometric sum expression

$$
Z_{m}(x):=\sum_{k=0}^{2 m}(-1)^{k}\left(\begin{array}{c}
2 m \\
k
\end{array}\right)^{3}\left(\begin{array}{c}
x+k \\
k
\end{array}\right) .
$$

With Zeilberger's paradigm of "creative telescoping" [25] we compute a linear recurrence for (6),

$$
a_{0}(x, m) Z_{m}(x)+a_{1}(x, m) Z_{m+1}(x)+a_{2}(x, m) Z_{m+2}(x)+a_{3}(x, m) Z_{m+3}(x)=0,
$$

with $a_{i}(x, m) \in \mathbb{Z}[x, m]$ and $a_{3}(x, m)$ not identically zero. In the next step we apply the differential operator $D$ to both sides of (7), which results in the mixed differential-difference equation

$$
\begin{aligned}
& a_{0}^{\prime}(x, m) Z_{m}(x)+a_{1}^{\prime}(x, m) Z_{m+1}(x)+a_{2}^{\prime}(x, m) Z_{m+2}(x)+a_{3}^{\prime}(x, m) Z_{m+3}(x) \\
& \quad+a_{0}(x, m) Z_{m}^{\prime}(x)+a_{1}(x, m) Z_{m+1}^{\prime}(x)+a_{2}(x, m) Z_{m+2}^{\prime}(x)+a_{3}(x, m) Z_{m+3}^{\prime}(x)=0 .
\end{aligned}
$$

After applying the operator $L$ to both sides, and using the fact that

$$
Z_{m}^{\prime}(0)=\sum_{k=0}^{2 m}(-1)^{k}\left(\begin{array}{c}
2 m \\
k
\end{array}\right)^{3} L D\left(\begin{array}{c}
x+k \\
k
\end{array}\right)=\sum_{k=0}^{2 m}(-1)^{k}\left(\begin{array}{c}
2 m \\
k
\end{array}\right)^{3} H_{k}=G_{m}
$$

we obtain

$$
\begin{aligned}
a_{0}^{\prime}(0, m) Z_{m}(0)+ & a_{1}^{\prime}(0, m) Z_{m+1}(0)+a_{2}^{\prime}(0, m) Z_{m+2}(0)+a_{3}^{\prime}(0, m) Z_{m+3}(0) \\
& +a_{0}(0, m) G_{m}+a_{1}(0, m) G_{m+1}+a_{2}(0, m) G_{m+2}+a_{3}(0, m) G_{m+3}=0 .
\end{aligned}
$$

The $a_{i}(0, m)$ are polynomials in $m$ and $a_{3}(0, m)$ is non-zero. (To avoid clutter these polynomials are not given here but they can be found in [23].) Applying Dixon's identity [8, Ex. 5.15],

$$
Z_{m}(0)=\sum_{k=0}^{2 m}(-1)^{k}\left(\begin{array}{c}
2 m \\
k
\end{array}\right)^{3}=\frac{(3 m) !(-1)^{m}}{m ! m ! m !}
$$


gives the recurrence

$$
a_{0}(0, m) G_{m}+a_{1}(0, m) G_{m+1}+a_{2}(0, m) G_{m+2}+a_{3}(0, m) G_{m+3}=q(m) \frac{(3 m) !(-1)^{m}}{m ! m ! m !}
$$

for some $q(m) \in \mathbb{Q}(m)$. Now it is an easy step to verify that the right-hand side of $(2)$ is indeed a solution of the recurrence (11). Checking the first initial values finishes the proof of identity (2).

We now turn to proofs of (1) and (3). As to the origin of (1), we note that one of the hardest challenges in [5] was proving the identity

$$
\sum_{k=0}^{m}(-1)^{k}\left(\begin{array}{c}
m \\
k
\end{array}\right)^{3}\left[3\left(H_{m-k}^{2}-H_{k}^{2}\right)+H_{m-k}^{(2)}+H_{k}^{(2)}\right]=0 .
$$

For odd $m$, this is easy to see by a simple symmetry argument: $k \longleftrightarrow m-k$ turns the sum into its own negative. So we concentrate on even $m$, for which we write $2 m$, and perform simple operations and thus are left with the identity (1).

Before we continue we point out that the Andrews-Newton-Zeilberger method can be modified by using the binomials already present in the sum. The chances of recognizing known identities and getting corollaries are higher this way. In this context differentiation formulas such as $\frac{d}{d x}\left(\begin{array}{c}m+x \\ k+x\end{array}\right)=\left(\begin{array}{c}m+x \\ k+x\end{array}\right)\left(H_{m+x}-H_{k+x}\right)$, followed by setting $x=0$, are useful.

Let us demonstrate this by a simple example: From Vandermonde's formula [8, p. 169], we have

$$
\sum_{k \geq i}\left(\begin{array}{c}
m-i \\
k-i
\end{array}\right)\left(\begin{array}{c}
m+x \\
k+x
\end{array}\right)=\left(\begin{array}{c}
2 m-i+x \\
m+x
\end{array}\right) .
$$

Hence, by the differentiation strategy alluded to above,

$$
\sum_{k \geq i}\left(\begin{array}{c}
m-i \\
k-i
\end{array}\right)\left(\begin{array}{c}
m \\
k
\end{array}\right)\left(H_{m}-H_{k}\right)=\left(\begin{array}{c}
2 m-i \\
m
\end{array}\right)\left(H_{2 m-i}-H_{m}\right) .
$$

Proofs of (1) and (3) are based on similar manipulations, based on the identity

$$
\sum_{k=0}^{2 m}(-1)^{k}\left(\begin{array}{c}
2 m+x \\
k
\end{array}\right)\left(\begin{array}{c}
2 m+x+y \\
x+k
\end{array}\right)\left(\begin{array}{c}
2 m+y \\
y+k
\end{array}\right)=\frac{(3 m+x+y) !(-1)^{m}}{m !(m+x) !(m+y) !}
$$

This seems to be the only identity useful in this context. Note that the special case $x=y=0$ is Dixon's formula (10).

Let us start with the special case $y=0$ :

$$
\sum_{k=0}^{2 m}(-1)^{k}\left(\begin{array}{c}
2 m+x \\
k
\end{array}\right)\left(\begin{array}{c}
2 m+x \\
x+k
\end{array}\right)\left(\begin{array}{c}
2 m \\
k
\end{array}\right)=\frac{(3 m+x) !(-1)^{m}}{m ! m !(m+x) !} .
$$

Differentiating (14), and setting $x=0$, one gets

$$
2 \sum_{k=0}^{2 m}(-1)^{k}\left(\begin{array}{c}
2 m \\
k
\end{array}\right)^{3} H_{k}=\frac{(3 m) !(-1)^{m}}{m ! m ! m !}\left[H_{m}+2 H_{2 m}-H_{3 m}\right],
$$

which is an independent proof of (2).

Differentiating (14) twice, and setting $x=0$, one gets 


$$
\begin{aligned}
& 2 \sum_{k=0}^{2 m}(-1)^{k}\left(\begin{array}{c}
2 m \\
k
\end{array}\right)^{3}\left[H_{k}^{(2)}+H_{k}^{2}+H_{k} H_{2 m-k}\right] \\
& \quad=\frac{(3 m) !(-1)^{m}}{m ! m ! m !}\left[-H_{3 m}^{(2)}+H_{m}^{(2)}+\left(H_{3 m}-H_{m}\right)^{2}+2 H_{2 m}^{(2)}+4 H_{2 m}^{2}-4\left(H_{3 m}-H_{m}\right) H_{2 m}\right] .
\end{aligned}
$$

If one differentiates (13) with respect to $x$ and $y$ and replaces both variables by zero, one gets

$$
\begin{aligned}
2 \sum_{k=0}^{2 m}(-1)^{k}\left(\begin{array}{c}
2 m \\
k
\end{array}\right)^{3}\left[H_{k}^{2}+H_{k} H_{2 m-k}\right] \\
\quad=\frac{(3 m) !(-1)^{m}}{m ! m ! m !}\left[\left(H_{3 m}-H_{m}\right)^{2}+H_{2 m}^{(2)}-H_{3 m}^{(2)}-4 H_{2 m}\left(H_{3 m}-H_{m}\right)+4 H_{2 m}^{2}\right] .
\end{aligned}
$$

Subtracting the last two equations, we find

$$
2 \sum_{k=0}^{2 m}(-1)^{k}\left(\begin{array}{c}
2 m \\
k
\end{array}\right)^{3} H_{k}^{(2)}=\frac{(3 m) !(-1)^{m}}{m ! m ! m !}\left[H_{m}^{(2)}+H_{2 m}^{(2)}\right]
$$

which is $(3)$.

The above computations evaluate part of the crucial identity (1), but one also needs a formula for

$$
C_{m}:=\sum_{k=0}^{2 m}(-1)^{k}\left(\begin{array}{c}
2 m \\
k
\end{array}\right)^{3}\left[H_{k}^{2}-H_{k}^{(2)}\right]
$$

and for this we have to resort to recurrences. Write

$$
A_{m}:=\sum_{k=0}^{2 m}(-1)^{k}\left(\begin{array}{c}
2 m \\
k
\end{array}\right)^{3}\left[H_{k}^{2}+H_{k} H_{2 m-k}\right], \quad B_{m}:=\sum_{k=0}^{2 m}(-1)^{k}\left(\begin{array}{c}
2 m \\
k
\end{array}\right)^{3} H_{k}^{(2)} .
$$

Both these quantities have already been evaluated, but $C_{m}$ is not yet known. Identity (1) holds if and only if $-3 A_{m}+7 B_{m}+6 C_{m}=0$ for all positive integers $m$, or in other words,

$$
C_{m}=\frac{1}{12} \frac{(3 m) !(-1)^{m}}{m ! m ! m !}\left[3\left(H_{m}+2 H_{2 m}-H_{3 m}\right)^{2}-7 H_{m}^{(2)}-4 H_{2 m}^{(2)}-4 H_{2 m}^{(2)}-3 H_{3 m}^{(2)}\right] .
$$

We will prove the identity (17) with the Andrews-Newton-Zeilberger method. Here the crucial observation is that

$$
L D^{2} Z_{m}(x)=C_{m}
$$

Hence, if we apply the differentiation operator $D$ to the recurrence (8) and then apply $L$ to the result, we obtain

$$
\begin{aligned}
& a_{0}^{\prime \prime}(0, m) Z_{m}(0)+a_{1}^{\prime \prime}(0, m) Z_{m+1}(0)+a_{2}^{\prime \prime}(0, m) Z_{m+2}(0, m)+a_{3}^{\prime \prime}(0, m) Z_{m+3}(0) \\
& \quad+2\left[a_{0}^{\prime}(0, m) Z_{m}^{\prime}(0)+a_{1}^{\prime}(0, m) Z_{m+1}^{\prime}(0)+a_{2}^{\prime}(0, m) Z_{m+2}^{\prime}(0, m)+a_{3}^{\prime}(0, m) Z_{m+3}^{\prime}(0)\right] \\
& \quad+a_{0}(0, m) Z_{m}^{\prime \prime}(0)+a_{1}(0, m) Z_{m+1}^{\prime \prime}(0)+a_{2}(0, m) Z_{m+2}^{\prime \prime}(0, m)+a_{3}(0, m) Z_{m+3}^{\prime \prime}(0)=0 .
\end{aligned}
$$


Together with $(2),(6),(10)$, and (18), and the fact that $H_{m+1}=H_{m}+\frac{1}{m+1}$, we obtain the recurrence

$$
\begin{aligned}
a_{0}(0, m) C_{m}+a_{1}(0, m) & C_{m+1}+a_{2}(0, m) C_{m+2}+a_{3}(0, m) C_{m+3} \\
& =\frac{(3 m) !(-1)^{m}}{m ! m ! m !}\left[q_{0}(m)+q_{1}(m) H_{m}+q_{2}(m) H_{2 m}+q_{3}(m) H_{3 m}\right]
\end{aligned}
$$

for some $q_{i}(m) \in \mathbb{Q}(m)$. By checking that the right-hand side of (17) is a solution of (20) and comparing the first initial values we have proved identity (17).

We find it fascinating that identity (1) and hence also identity (12) can be proved with purely hypergeometric tools following the Andrews-Newton-Zeilberger method. Nevertheless, this strategy may not always be successful. In the above example it is crucial that one can find closed form evaluations for the sums $A_{m}$ and $B_{m}$. For example, so far we have not succeeded in proving

$$
\begin{aligned}
& \sum_{k=0}^{m}\left[3\left(4\left(H_{m-k}-H_{k}\right)^{2}+H_{m-k}^{(2)}+H_{k}^{(2)}\right)\right. \\
& \left.\quad+2 k\left(8\left(H_{m-k}-H_{k}\right)^{3}+6\left(H_{m-k}-H_{k}\right)\left(H_{m-k}^{(2)}+H_{k}^{(2)}\right)+H_{m-k}^{(3)}-H_{k}^{(3)}\right)\right]\left(\begin{array}{c}
m \\
k
\end{array}\right)^{4}=0
\end{aligned}
$$

with the Andrews-Newton-Zeilberger method, which was one of the identities required in [24]. In contrast, in [5] both identities (12) and (21) were proved with the symbolic summation package Sigma $[20,18]$. It computes for (12) the recurrence

$$
3(2+3 m)(4+3 m) T_{m}+(2+m)^{2} T_{2+m}=0
$$

and therefore proves the identity once the first two initial values have been established. Similarly, for (21) Sigma computed a homogeneous recurrence of order three [23].

The approach based on the Sigma package is completely different from the Andrews-NewtonZeilberger method. Nested sum expressions, including summations involving harmonic numbers, are translated in a natural way into the corresponding difference field setting of $\Pi \Sigma$-fields $[4,10,11,18]$, and, by using a very general algebraic machinery $[4,22,19,21]$, the problem is solved there. In the Sigma package a streamlined version of Karr's indefinite summation algorithm [10] is implemented which is in a sense the summation counterpart to Risch's algorithm $[15,16]$ for indefinite integration. Moreover, the summation package Sigma contains several generalizations that enables one to deal also with definite multisum identities.

Coming to a similar conclusion as in [12], the Sigma approach provides much more flexibility and efficiency when dealing with definite nested sum expressions due to the richness of the underlying algebraic structure. These aspects will be illustrated further in the next section.

\section{Finding Closed form evaluations With Sigma}

In this section we show how the package Sigma may be used not only to prove identities, but also to discover them. We demonstrate this by finding and proving the following two 
identities

$$
\begin{aligned}
& \sum_{k=0}^{2 m}(-1)^{k}\left(\begin{array}{c}
2 m \\
k
\end{array}\right)^{3} H_{k} H_{2 m-k}=\frac{(3 m) !(-1)^{m}}{m ! m ! m !} \\
& \times \frac{1}{12}\left(3 H_{m}^{2}+12 H_{2 m}\left(H_{2 m}+H_{m}-H_{3 m}\right)-6 H_{m} H_{3 m}+3 H_{3 m}^{2}+H_{m}^{(2)}+4 H_{2 m}^{(2)}-3 H_{3 m}^{(2)}\right)
\end{aligned}
$$

and

$$
\begin{aligned}
& \sum_{k=0}^{2 m}(-1)^{k}\left(\begin{array}{c}
2 m \\
k
\end{array}\right)^{3} H_{k}^{2}=\frac{(3 m) !(-1)^{m}}{m ! m ! m !} \\
& \times \frac{1}{12}\left(3 H_{m}^{2}+12 H_{2 m}\left(H_{2 m}+H_{m}-H_{3 m}\right)-6 H_{m} H_{3 m}+3 H_{3 m}^{2}-H_{m}^{(2)}+2 H_{2 m}^{(2)}-3 H_{3 m}^{(2)}\right) .
\end{aligned}
$$

Once we have established these two identities, along with (15), we have therefore discovered/constructed the desired identity (1).

We follow the paradigm introduced in [14], but generalize it from hypergeometric summation problems to nested multisum problems. First one tries to compute a recurrence with creative telescoping and then one attempts to solve the recurrence in terms of nested sum expressions. Both steps can be done completely automatically.

In the following we illustrate how one can find a closed form evaluation of the left side of (23), which we call $S_{m}$. We start the Mathematica session by loading the package Sigma with

$\ln [1]:=<<$ Sigma $^{6}$

$$
\text { Sigma - A summation package by Carsten Schneider (C) RISC-Linz }
$$

Then we set up the summation problem as follows:

$$
\begin{aligned}
& \operatorname{In}[2]:=\text { mySum }=\text { SigmaSum }[ \\
& \left.\operatorname{SigmaHNumber}[\mathbf{k}]^{\wedge} \text { 2SigmaBinomial }[\mathbf{m}, \mathbf{k}]^{\wedge} \text { 3SigmaPower }[-\mathbf{1}, \mathbf{k}],\{\mathbf{k}, \mathbf{0}, \mathbf{2 m}\}\right] \\
& \operatorname{Out}[2]=\sum_{\mathrm{k}=0}^{2 \mathrm{~m}} \mathrm{H}_{\mathrm{k}}^{2}\left(\left(\begin{array}{l}
\mathrm{m} \\
\mathrm{k}
\end{array}\right)\right)^{3}(-1)^{\mathrm{k} .}
\end{aligned}
$$

Remark. The basic functions SigmaSum and SigmaProduct are used to describe all nested sum and product expressions that can be formulated in $\Pi \Sigma$-fields. Further details about this difference field machinery and the summation theory can be found in $[4,10,11,18]$. To facilitate this task there are numerous other functions available, like SigmaHNumber, SigmaBinomial or SigmaPower. For instance, SigmaHNumber $[\mathrm{k}]$ produces the $k$ th harmonic number $H_{k}$ which alternatively could be described by SigmaSum $[1 / i,\{i, 1, k\}]$.

Finding a recurrence. As already mentioned above, we first try to compute a recurrence for the definite sum mySum. To compute such recurrences Zeilberger's creative telescoping [25] has been extended from hypergeometric expressions to terms in $\Pi \Sigma$-fields; for more information see [18]. In this instance we succeed in computing a recurrence of order four

$$
a_{0}(m) S_{m}+a_{1}(m) S_{m+1}+a_{2}(m) S_{m+2}+a_{3}(m) S_{m+3}+a_{4}(m) S_{m+4}=0,
$$

with polynomial coefficients $a_{i}(m) \in \mathbb{Z}[m]$ and $a_{4}(m)$ non-zero. (We failed to find a recurrence of smaller order.) Note that the computation time is relatively costly and the integer coefficients of the polynomials $a_{i}(m)$ become quite large. We could go on, as mentioned above, to solve the recurrence but the solutions are unwieldy. Combining these solutions would lead to a closed form evaluation for $S_{m}$. 
But we can do it much better. Schneider's summation theory provides a new mechanism, illustrated in [12], which finds certain sum extensions automatically. The details of this method are described in [18], so we restrict ourselves to a brief description of its application. This mechanism is activated by setting the option SimplifyByExt $\rightarrow$ DepthNumber.

$$
\begin{aligned}
& \ln [3]:=\text { rec }=\text { GenerateRecurrence[mySum, SimplifyByExt } \rightarrow \text { DepthNumber] } \\
& \text { Out }[3]=\left\{-108\left(9\left(2+9 m+9 m^{2}\right)^{2}\left(378+1508 m+1999 m^{2}+1098 m^{3}+216 m^{4}\right) \operatorname{SUM}[m]+\right.\right. \\
& (1+\mathrm{m})\left(3 \left(2316+21420 \mathrm{~m}+77561 \mathrm{~m}^{2}+145192 \mathrm{~m}^{3}+153369 \mathrm{~m}^{4}+92250 \mathrm{~m}^{5}+29484 \mathrm{~m}^{6}\right.\right. \\
& \left.\left.\left.+3888 \mathrm{~m}^{7}\right) \operatorname{SUM}[1+\mathrm{m}]+(2+\mathrm{m})^{3}\left(39+284 \mathrm{~m}+685 \mathrm{~m}^{2}+666 \mathrm{~m}^{3}+216 \mathrm{~m}^{4}\right) \operatorname{SUM}[2+\mathrm{m}]\right)\right)== \\
& \frac{27}{4(1+m)^{2}(2+m)(1+2 m)(3+2 m)^{2}}\left(-2\left(3737160+62577798 m+462384333 m^{2}+1992022405 m^{3}\right.\right. \\
& +5577688302 m^{4}+10708407249 m^{5}+14477150099 m^{6}+13910415390 m^{7}+9444730896 m^{8} \\
& \left.+4427405568 \mathrm{~m}^{9}+1362728448 \mathrm{~m}^{10}+247743360 \mathrm{~m}^{11}+20155392 \mathrm{~m}^{12}\right) \sum_{\iota_{1}=0}^{2 \mathrm{~m}}\left(\left(\begin{array}{c}
2 \mathrm{~m} \\
\iota_{1}
\end{array}\right)\right)^{3}(-1)^{\iota_{1}} . \\
& +\left(356818+4707387 \mathrm{~m}+27350433 \mathrm{~m}^{2}+92529298 \mathrm{~m}^{3}+202786961 \mathrm{~m}^{4}\right. \\
& +302606531 m^{5}+313999848 m^{6}+226727820 m^{7}+111716496 m^{8}+35798112 m^{9} \\
& \left.\left.\left.+6718464 \mathrm{~m}^{10}+559872 \mathrm{~m}^{11}\right)\left(-1+(1+2 \mathrm{~m})^{3} \sum_{\iota_{1}=0}^{2 \mathrm{~m}} \frac{\left(\left(2 \mathrm{~m} \iota_{1}\right)\right)^{3}(-1)^{\iota_{1}}}{\left(1+2 \mathrm{~m}-\iota_{1}\right)^{3}}\right)\right)\right\}
\end{aligned}
$$

This means that SUM $[\mathrm{m}]=S_{m}$ (= mySum) satisfies the output recurrence rec. We emphasize that we found two sum extensions automatically, which allowed us to compute an inhomogeneous recurrence of order two, instead of a homogeneous recurrence of order four as in the standard approach (cf. (24)). In particular, the recurrence formula is much less complicated and the computation time has been reduced by about 90 percent.

Applying Dixon's identity (10), as well as

$$
\sum_{k=0}^{2 m} \frac{(-1)^{k}\left(\begin{array}{c}
2 m \\
k
\end{array}\right)^{3}}{(2 m-k+1)^{3}}=\frac{1}{(2 m+1)^{3}}
$$

(which can be deduced from (13) or from a straightforward application of Zeilberger's algorithm), this recurrence can be simplified further to a recurrence where the inhomogeneous part is just a hypergeometric expression:

$$
\begin{aligned}
& \ln [4]:=\text { rec }=\left\{-108\left(9 ( 2 + 9 \mathbf { m } + 9 \mathbf { m } ^ { 2 } ) ^ { 2 } \left(378+1508 \mathbf{m}+1999 \mathbf{m}^{2}+1098 \mathbf{m}^{3}\right.\right.\right. \\
& \left.+216 \mathbf{m}^{4}\right) \mathrm{SUM}[\mathrm{m}]+(1+\mathbf{m})\left(3 \left(2316+21420 \mathrm{~m}+77561 \mathbf{m}^{2}+145192 \mathbf{m}^{3}+\right.\right. \\
& \left.153369 \mathbf{m}^{4}+92250 \mathbf{m}^{5}+29484 \mathbf{m}^{6}+3888 \mathbf{m}^{7}\right) \operatorname{SUM}[1+\mathbf{m}]+(2+\mathbf{m})^{3} \\
& \left.\left.\left(39+284 \mathrm{~m}+685 \mathrm{~m}^{2}+666 \mathrm{~m}^{3}+216 \mathrm{~m}^{4}\right) \operatorname{SUM}[2+\mathrm{m}]\right)\right)== \\
& -\left(2 7 \left(1245720+20028786 \mathrm{~m}+140775587 \mathrm{~m}^{2}+570157077 \mathrm{~m}^{3}+1479124716 \mathrm{~m}^{4}\right.\right. \\
& +2583385939 m^{5}+3103459407 m^{6}+2567832192 m^{7}+1436355504 m^{8} \\
& \left.\left.+518231520 \mathbf{m}^{9}+108755136 \mathbf{m}^{10}+10077696 \mathbf{m}^{11}\right)(3 \mathbf{m}) !(-1)^{\mathrm{m} \cdot}\right) / \\
& \left.\left(\mathbf{2}(\mathbf{1}+\mathbf{m})^{2}(\mathbf{2}+\mathbf{m})(\mathbf{1}+\mathbf{2} \mathbf{m})(\mathbf{3}+\mathbf{2} \mathbf{m}) \mathbf{m} !^{\mathbf{3}}\right)\right\}
\end{aligned}
$$

Solving the recurrence. Since this simplified version of the recurrence lies in the input class of the recurrence-solver of Sigma, we construct a solution with:

$\ln [5]:=\operatorname{recSol}=$ SolveRecurrence $[\operatorname{rec}[[1]], \mathbf{S U M}[\mathbf{n}]]$ 
Out $[5]=\left\{\left\{0, \frac{(3 \mathrm{~m}) !(-1)^{\mathrm{m}} \cdot}{\mathrm{m} !^{3}}\right\}\right\}$

As a result we find the solution $\frac{(3 m) !(-1)^{m}}{m !^{3}}$ of the homogeneous version of the recurrence rec. The solver fails, however, to find a solution of the inhomogeneous recurrence itself. This tells us that the underlying difference field $\mathbb{Q}\left(m, \frac{(-1)^{m}(3 m) !}{m !^{3}}\right)$, which we constructed automatically by the objects given in the recurrence rec, is too small. By setting the option Extension $\rightarrow$ SUMS we find all nested sum solutions expressed in terms of objects given in the recurrence.

$\ln [6]:=\operatorname{recSol}=$ SolveRecurrence $[\operatorname{rec}[[1]], \mathbf{S U M}[\mathbf{m}]$, Extension $\rightarrow$ SUMS $]$

$$
\begin{aligned}
\text { Out }[6]=\left\{\left\{0, \frac{(3 \mathrm{~m}) ! \cdot(-1)^{\mathrm{m}} \cdot}{\mathrm{m} !^{3}}\right\},\left\{0, \frac{(3 \mathrm{~m}) ! \cdot(-1)^{\mathrm{m} \cdot} \sum_{\iota_{1}=1}^{\mathrm{m}} \frac{-10+68 \iota_{1}-153 \iota_{1}^{2}+108 \iota_{1}^{3}}{\iota_{1}\left(-2+13 \iota_{1}-27 \iota_{1}^{2}+18 \iota_{1}^{3}\right)}}{\mathrm{m} !^{3}}\right\}\right. \\
\left\{1, \frac{1}{72 \mathrm{~m} !^{3}}\left(( 3 \mathrm { m } ) ! \cdot ( - 1 ) ^ { \mathrm { m } \cdot } \sum _ { \iota _ { 1 } = 1 } ^ { \mathrm { m } } \left(-220+22839 \iota_{1}^{2}-167482 \iota_{1}^{3}+546174 \iota_{1}^{4}-935487 \iota_{1}^{5}+819558 \iota_{1}^{6}\right.\right.\right. \\
-289656 \iota_{1}^{7}+4 \iota_{1}\left(20-266 \iota_{1}+1460 \iota_{1}^{2}-4221 \iota_{1}^{3}+6759 \iota_{1}^{4}-5670 \iota_{1}^{5}+1944 \iota_{1}^{6}\right) \\
\left.\left.\left.\left.\quad \sum_{\iota_{2}=1} \frac{-10+68 \iota_{2}-153 \iota_{2}^{2}+108 \iota_{2}^{3}}{\iota_{2}\left(-2+13 \iota_{2}-27 \iota_{2}^{2}+18 \iota_{2}^{3}\right)}\right) /\left(\left(1-3 \iota_{1}\right)^{2}\left(2-3 \iota_{1}\right)^{2}\left(1-2 \iota_{1}\right)^{2} \iota_{1}^{2}\right)\right)\right\}\right\}
\end{aligned}
$$

We therefore succeeded in finding all solutions of our recurrence: the first two entries give us two linearly independent solutions of the homogeneous version of the recurrence and the third entry is a particular solution of the inhomogeneous recurrence. These nested sum solutions are included in the class of d'Alembertian solutions, which are introduced in [1]; further results can be found in $[9,18]$.

Combining the solutions. In the end, the closed form of mySum is that particular linear combination of the homogeneous solutions plus the inhomogeneous solution that matches the initial values of mySum.

$$
\begin{aligned}
\ln [7]:= & \text { result }=\text { FindLinearCombination }[\text { recSol, mySum, } 2] \\
\text { Out }[7]= & \frac{(3 \mathrm{~m}) !(-1)^{\mathrm{m}} .}{72 \mathrm{~m} !^{3}}\left(149 \sum_{\iota_{1}=1}^{\mathrm{m}} \frac{-10+68 \iota_{1}-153 \iota_{1}^{2}+108 \iota_{1}^{3}}{\iota_{1}\left(-2+13 \iota_{1}-27 \iota_{1}^{2}+18 \iota_{1}^{3}\right)}+\right. \\
& \sum_{\iota_{1}=1}^{\mathrm{m}}\left(-220+22839 \iota_{1}^{2}-167482 \iota_{1}^{3}+546174 \iota_{1}^{4}-935487 \iota_{1}^{5}+819558 \iota_{1}^{6}\right. \\
& -289656 \iota_{1}^{7}+4 \iota_{1}\left(20-266 \iota_{1}+1460 \iota_{1}^{2}-4221 \iota_{1}^{3}+6759 \iota_{1}^{4}-5670 \iota_{1}^{5}+1944 \iota_{1}^{6}\right) \\
& \left.\left.\left.\sum_{\iota_{2}=1}^{\iota_{1}} \frac{-10+68 \iota_{2}-153 \iota_{2}^{2}+108 \iota_{2}^{3}}{\iota_{2}\left(-2+13 \iota_{2}-27 \iota_{2}^{2}+18 \iota_{2}^{3}\right)}\right) /\left(\left(1-3 \iota_{1}\right)^{2}\left(2-3 \iota_{1}\right)^{2}\left(1-2 \iota_{1}\right)^{2} \iota_{1}^{2}\right)\right)\right)
\end{aligned}
$$

Simplification with indefinite summation. So far we found a closed form evaluation of mySum in terms of a two-nested sum. Now it is a major task to simplify this expression so that it contains single-nested sums only, like mySum. This cannot be achieved by Gosper's algorithm [7] since the innermost sum is not Gosper-summable; or in other words, it cannot be expressed by a linear combination of hypergeometric terms. But fortunately our indefinite summation algorithm can deal with such nested multisum expressions. In this instance we are able to find a single-nested sum automatically in order to eliminate the outermost summation quantifier of the two-nested sum in result. This can be achieved with the following function call provided by Sigma. 


$$
\begin{aligned}
& \operatorname{In}[8]:= \text { result }=\text { SigmaReduce }[\text { result }, \mathbf{m}, \text { SimplifyByExt } \rightarrow \text { Depth }] \\
& \text { Out }[8]=\frac{(3 \mathrm{~m}) ! .(-1)^{\mathrm{m}} \cdot}{72 \mathrm{~m} !^{3}}\left(149 \sum_{\iota_{1}=1}^{\mathrm{m}} \frac{-10+68 \iota_{1}-153 \iota_{1}^{2}+108 \iota_{1}^{3}}{\iota_{1}\left(-2+13 \iota_{1}-27 \iota_{1}^{2}+18 \iota_{1}^{3}\right)}+2\left(\sum_{\iota_{1}=1}^{\mathrm{m}} \frac{-10+68 \iota_{1}-153 \iota_{1}^{2}+108 \iota_{1}^{3}}{\iota_{1}\left(-2+13 \iota_{1}-27 \iota_{1}^{2}+18 \iota_{1}^{3}\right)}\right)^{2}-\right. \\
& \\
&\left.\sum_{\iota_{1}=1}^{\mathrm{m}} \frac{20+2720 \iota_{1}-38207 \iota_{1}^{2}+213418 \iota_{1}^{3}-622368 \iota_{1}^{4}+1001583 \iota_{1}^{5}-842886 \iota_{1}^{6}+289656 \iota_{1}^{7}}{\left(1-3 \iota_{1}\right)^{2}\left(2-3 \iota_{1}\right)^{2}\left(1-2 \iota_{1}\right)^{2} \iota_{1}^{2}}\right)
\end{aligned}
$$

Furthermore, by setting the option SimpleSumRepresentation $\rightarrow$ True, the sum extension is expressed in terms of simpler sums:

$$
\begin{aligned}
& \operatorname{In}[9]:=\text { result }=\text { SigmaReduce }[\text { result, } \mathbf{m} \text {, SimpleSumRepresentation } \rightarrow \text { True }] \\
& \text { Out }[9]=\frac{(3 \mathrm{~m}) ! \cdot(-1)^{\mathrm{m}}}{72 \mathrm{~m} !^{3}}\left(-18 \sum_{\iota_{1}=1}^{\mathrm{m}} \frac{1}{\left(1-3 \iota_{1}\right)^{2}}-18 \sum_{\iota_{1}=1}^{\mathrm{m}} \frac{1}{\left(2-3 \iota_{1}\right)^{2}}+12 \sum_{\iota_{1}=1}^{\mathrm{m}} \frac{1}{\left(1-2 \iota_{1}\right)^{2}}-5 \sum_{\iota_{1}=1}^{\mathrm{m}} \frac{1}{\iota_{1}^{2}}+2\right. \\
& \left.\left.\qquad\left(5 \sum_{\iota_{1}=1}^{\mathrm{m}} \frac{1}{\iota_{1}}+6 \sum_{\iota_{1}=1}^{\mathrm{m}} \frac{1}{-1+2 \iota_{1}}-3\left(\sum_{\iota_{1}=1}^{\mathrm{m}} \frac{1}{-2+3 \iota_{1}}+\sum_{\iota_{1}=1}^{\mathrm{m}} \frac{1}{-1+3 \iota_{1}}\right)\right)\right)^{2}\right)
\end{aligned}
$$

Finally, we notice that these sum extensions can be expressed in terms of harmonic numbers. Our indefinite summation algorithms finds this closed form evaluation by building up the underlying difference field with the manually given extensions $H_{m}, H_{2 m}, H_{m}^{(2)}, H_{2 m}^{(2)}, H_{3 m}$ and $H_{3 m}^{(2)}$.

$\ln [10]:=$ SigmaReduce $\left[\right.$ result, $\mathbf{m}$, Tower $\left.\rightarrow\left\{\mathbf{H}_{\mathbf{m}}, \mathbf{H}_{\mathbf{2 m}}, \mathbf{H}_{\mathbf{m}}^{(2)}, \mathbf{H}_{\mathbf{2 m}}^{(2)}, \mathbf{H}_{\mathbf{3 m}}, \mathbf{H}_{\mathbf{3 m}}^{(2)}\right\}\right]$

Out $\left.[10]=\frac{(3 \mathrm{~m}) ! \cdot(-1)^{\mathrm{m}}}{12 \mathrm{~m} !^{3}}\left(3 \mathrm{H}_{\mathrm{m}}^{2}+12 \mathrm{H}_{2 \mathrm{~m}}\left(\mathrm{H}_{2 \mathrm{~m}}+\mathrm{H}_{\mathrm{m}}-\mathrm{H}_{3 \mathrm{~m}}\right)-6 \mathrm{H}_{\mathrm{m}} \mathrm{H}_{3 \mathrm{~m}}+3 \mathrm{H}_{3 \mathrm{~m}}^{2}-\mathrm{H}_{\mathrm{m}}^{(2)}+2 \mathrm{H}_{2 \mathrm{~m}}^{(2)}-3 \mathrm{H}_{3 \mathrm{~m}}^{(2)}\right)\right)$

Similar to identity (23), we succeed in finding the right-hand sides in the identities (16) and (22). This enables us to find or even to discover the identity (1), which was our aim. As illustrated above, however, the computations are relatively costly. In particular, to compute the recurrences for the given definite summation problems is a complicated task. As it turns out, in [5] we proved (12) without using the symmetry simplifications that led to (1), and discovered that it is a much simpler procedure than the one outlined here. Which strategy is better one cannot judge before actually getting involved in the computations.

\section{Other IDENTities AND REPEATED DEFinite SUMMATION}

In the literature combinatorial identities involving third powers of the binomial coefficient $\left(\begin{array}{c}m \\ k\end{array}\right)$ are relatively rare. The only examples known to us are Dixon's identity (10), and MacMahon's identity [6, p. 177 (5.107)]

$$
\sum_{k=0}^{2 m} x^{k} y^{2 m-k}\left(\begin{array}{c}
2 m \\
k
\end{array}\right)^{3}=\sum_{k=0}^{m} x^{k} y^{k}(x+y)^{2 m-2 k}\left(\begin{array}{c}
2 m \\
2 k
\end{array}\right)\left(\begin{array}{c}
2 k \\
k
\end{array}\right)\left(\begin{array}{c}
2 m+k \\
k
\end{array}\right) .
$$

We apply the results of this paper to this identity to generate new ones.

From MacMahon's identity we get

$$
\sum_{k=0}^{2 m}(-x)^{k}\left(\begin{array}{c}
2 m \\
k
\end{array}\right)^{3}=\sum_{k=0}^{m}(-x)^{k}(1-x)^{2 m-2 k} \frac{(2 m+k) !}{(2 m-2 k) ! k ! k ! k !} .
$$


Now we replace $x^{k}$ in this polynomial identity by $H_{k}$; this technique belongs to realm of the umbral calculus [17]. For the left-hand side one obtains

$$
\sum_{k=0}^{2 m}(-1)^{k}\left(\begin{array}{c}
2 m \\
k
\end{array}\right)^{3} H_{k}=\frac{(3 m) !(-1)^{m}}{2 m ! m ! m !}\left[-H_{3 m}+H_{m}+2 H_{2 m}\right],
$$

which is formula (2). For the right-hand side we compute

$$
x^{k}(1-x)^{2 m-2 k}=\sum_{j=0}^{2 m-2 k}(-1)^{j}\left(\begin{array}{c}
2 m-2 k \\
j
\end{array}\right) x^{j+k}
$$

and again replacing $x^{k}$ by $H_{k}$ gives

$$
\sum_{j=0}^{2 m-2 k}(-1)^{j}\left(\begin{array}{c}
2 m-2 k \\
j
\end{array}\right) H_{j+k}=-\frac{(2 m-2 k) ! k !}{(2 m-k) !(2 m-2 k)}, \quad k \neq m
$$

after simplification. (This identity was proved as an introductory example in [5].) In the exceptional case $k=m$ the right-hand side simplifies to $H_{k}$. Thus

$$
\frac{(3 m) !(-1)^{m}}{2 m ! m ! m !}\left[-H_{3 m}+H_{m}+2 H_{2 m}\right]=\sum_{k=0}^{m-1}(-1)^{k-1} \frac{(2 m+k) !}{(2 m-k) ! k ! k !(2 m-2 k)}+\frac{(3 m) !(-1)^{m} H_{m}}{m ! m ! m !},
$$

which yields the noteworthy identity

$$
\sum_{k=0}^{m-1}(-1)^{k} \frac{(2 m+k) !}{(2 m-k) ! k ! k !(2 m-2 k)}=\frac{(3 m) !(-1)^{m}}{2 m ! m ! m !}\left[H_{m}-2 H_{2 m}+H_{3 m}\right] .
$$

On the left-hand side, one might ask what happens if one sums over the "full" range $k=$ $0, \ldots, 2 m$, with the exception of the term $k=m$. Indeed, one has, due to a standard proof by Zeilberger's algorithm,

$$
\sum_{k=0}^{2 m}(-1)^{k} \frac{(2 m+k) !}{(2 m-k) ! k ! k ! 2(z-k)}=\frac{(2 m+z) !(z-2 m-1) !}{2 z ! z !}
$$

and therefore

$$
\begin{aligned}
\sum_{k=m+1}^{2 m}(-1)^{k} \frac{(2 m+k) !}{(2 m-k) ! k ! k ! 2(m-k)} & =\lim _{z \rightarrow m}\left[\frac{(2 m+z) !(z-2 m-1) !}{2 z ! z !}\right. \\
& \left.-\frac{(3 m) !(-1)^{m}}{2 m ! m ! m !}\left[H_{m}-2 H_{2 m}+H_{3 m}\right]-\frac{(3 m) !(-1)^{m}}{m ! m ! m ! 2(z-m)}\right] .
\end{aligned}
$$

Taking the limit on the right, we find the companion identity

$$
\sum_{k=m+1}^{2 m}(-1)^{k} \frac{(2 m+k) !}{(2 m-k) ! k ! k !(2 m-2 k)}=\frac{(3 m) !(-1)^{m}}{m ! m ! m !}\left[H_{2 m}-H_{m}\right] .
$$

Now it is a natural problem to consider and prove that for $|j| \leq m$

$$
\sum_{k=0}^{m-j-1}(-1)^{k} \frac{(2 m+k) !}{(2 m-k) ! k ! k ! 2(m-j-k)}=\frac{(3 m-j) !(-1)^{m-j}}{2(m-j) !^{2}(m+j) !}\left[H_{3 m-j}+H_{m+j}-2 H_{2 m}\right]
$$


which is (4), and its companion

$$
\sum_{k=m-j+1}^{2 m}(-1)^{k} \frac{(2 m+k) !}{(2 m-k) ! k ! k ! 2(m-j-k)}=\frac{(3 m-j) !(-1)^{m-j}}{(m-j) !^{2}(m+j) !}\left[H_{2 m}-H_{m-j}\right] .
$$

We denote the left-hand side of $(27)$ by $F_{j}(m)$, and compute a recurrence with shifts in $j$

$$
\begin{aligned}
-(j-m)^{2} F_{j}(m)+(j-3 m)(1+ & j+m) F_{j+1}(m) \\
& =-\frac{(-1)^{-j+m}(1+2 j-2 m)(-j+m)^{2}(-j+3 m) !}{2(1+j+m)(-j+3 m)(-j+m) !^{2}(j+m) !} .
\end{aligned}
$$

This can be achieved by Sigma or with any other algorithm that can deal with definite hypergeometric sums, like for instance [13]. Next, we solve this recurrence. The solution

$$
\frac{(-1)^{j}(-j+3 m) !}{(-j+m) !^{2}(j+m) !}
$$

of the homogeneous version of the recurrence is immediate. Moreover, by variation of the constant one obtains the particular solution

$$
\frac{(-1)^{j+m}(-j+3 m) !}{2(-j+m) !^{2}(j+m) !} \sum_{i=0}^{j} \frac{1+2 m-2 i}{(1+3 m-i)(m+i)} .
$$

Remark. We should mention that we failed to deal with this problem in our first attempt: First, we computed a recurrence with shifts in $m$ and not in $j$, namely

$$
\begin{aligned}
& 2(-1)^{j}(1+m)(1+j+m)(1+2 m)(-j+m) !^{2}(j+m) ! \\
& \left((-2+j-3 m)(-1+j-3 m)(-3+j-3 m) F_{j}(m)-(1-j+m)^{2}(1+j+m) F_{j}(m+1)\right) \\
& \quad=(-1)^{m}\left(j^{4}(3+4 m)-j^{3}\left(13+38 m+28 m^{2}\right)-(1+m)^{2}(1+m(8+9 m))+\right. \\
& \left.2 j^{2}\left(6+m\left(34+57 m+30 m^{2}\right)\right)-j(1+m)(-3+m(7+6 m(7+6 m)))\right)(-j+3 m) !
\end{aligned}
$$

Next, we tried to find all solutions as we tried it for the recurrence (29). But we failed to represent the particular solution of the inhomogeneous recurrence, like (31), since for any chosen lower summation bound we run into pole problems.

Given the solutions (30) and (31) to (29), we try to find a constant $c(m)$ (independent of $j$ ) such that

$$
F_{j}(m)=c(m) \frac{(-1)^{j}(-j+3 m) !}{(-j+m) !^{2}(j+m) !}+\frac{(-1)^{j+m}(-j+3 m) ! \sum_{i=0}^{j} \frac{1+2 m-2 i}{(1+3 m-i)(m+i)}}{2(-j+m) !^{2}(j+m) !} .
$$

This expression satisfies (29) identically. In order to compute $c(m)$, we set $j=0$ and find

$$
c(m)=\frac{-(-1)^{m}(1+2 m)(3 m) !+2 m(1+3 m) m !^{3} F_{0}(m)}{2 m(1+3 m)(3 m) !} .
$$

Note that we have simplified the problem from computing a closed form expression for $F_{j}(m)$, for general $j$, to evaluating only $F_{0}(m)$. Now $F_{0}(m)$ has, in fact, been evaluated in closed form in (25) above, so technically we are done. To show that everything can be done automatically, however, we shall rederive the formula for $F_{0}(m)$ here using mechanical summation tools. 
First, we compute an inhomogeneous recurrence of order one, namely

$$
\begin{aligned}
2(1+m)(1+2 m) m !^{3}\left[3(1+3 m)(2+3 m) F_{0}(m)+(1+m)^{2}\right. & \left.F_{0}(1+m)\right] \\
& =(-1)^{m}\left(1+8 m+9 m^{2}\right)(3 m) ! .
\end{aligned}
$$

(Note that this is a recurrence in the variable $m$, as opposed to (29) which is a recurrence in $j$.) Next, we solve this recurrence by variation of the constant and obtain, respectively,

$$
\frac{(-1)^{m}(3 m) !}{m !^{3}} \text { and } \frac{(-1)^{m-1}(3 m) !}{6 m !^{3}} \sum_{i=1}^{m} \frac{9 i^{2}-10 i+2}{i(2 i-1)(3 i-1)(3 i-2)}
$$

as the homogeneous and the particular solutions. Looking at the first initial value gives

$$
F_{0}(m)=\frac{(-1)^{m-1}(3 m) !}{6 m !^{3}} \sum_{i=1}^{m} \frac{9 i^{2}-10 i+2}{i(2 i-1)(3 i-1)(3 i-2)} .
$$

In other words, we managed to find a simpler form for $F_{0}(m)$, in the sense that it is an indefinite rational sum expression. By contrast, we started with a definite hypergeometric sum, cf. the left side of (25). Plugging this formula for $F_{0}(m)$ into (33), gives the closed form evaluation

$$
\begin{aligned}
F_{j}(m)=\frac{(3 m-j) !(-1)^{m-j}}{2(m-j) ! 2(m+j) !}\left[\sum_{i=0}^{j} \frac{1+2 m-2 i}{(1}+3 m-i\right)(m+i) \\
\left.\quad-\frac{1}{3} \sum_{i=1}^{m} \frac{9 i^{2}-10 i+2}{i(2 i-1)(3 i-1)(3 i-2)}-\frac{2 m+1}{m(3 m+1)}\right] .
\end{aligned}
$$

The two indefinite rational sums in this expression can be rephrased in terms of $H_{3 m-j}, H_{m+j}$ and $H_{2 m}$. This rewriting can be carried out automatically by Sigma (as we saw in Section 3). This finally leads to the identity (27), and hence (4).

In a slightly different fashion we were able to discover the companion identity (28). Alternatively, it could have been obtained by (26) and the difference with (27), and taking a limit.

Summarizing, all these computations can be carried out by Sigma in a completely mechanical way and hence can be fully automated. Moreover, we want to emphasize that the simplification strategy of definite summation, namely, (1) computing a recurrence, (2) solving the recurrence, and (3) combining those solutions, needed to be applied twice in order to find a closed form evaluation. We have never encountered a problem that required this sequence of operations more than once.

\section{REFERENCES}

[1] S. A. Abramov and M. Petkovšek. D'Alembertian solutions of linear differential and difference equations. In J. von zur Gathen, editor, Proc. ISSAC'94, pages 169-174. ACM Press, Baltimore, 1994.

[2] G. E. Andrews. Applications of basic hypergeometric functions. SIAM Rev., 16:441-484, 1974.

[3] G. E. Andrews and K. Uchimura. Identities in combinatorics. IV. Differentiation and harmonic numbers. Utilitas Math., 28:265-269, 1985.

[4] M. Bronstein. On solutions of linear ordinary difference equations in their coefficient field. J. Symbolic Comput., 29(6):841-877, June 2000. 
[5] K. Driver, H. Prodinger, C. Schneider, and J. A. C Weideman. Padé approximations to the logarithm II: Identities, recurrences, and symbolic computation. Ramanujan Journal, 11(2), 2006.

[6] G. P. Egorychev. Integral Representation and the Computation of Combinatorial Sums. American Mathematical Society, 1984.

[7] R. W. Gosper. Decision procedures for indefinite hypergeometric summation. Proc. Nat. Acad. Sci. U.S.A., 75:40-42, 1978.

[8] R. L. Graham, D. E. Knuth, and O. Patashnik. Concrete Mathematics: a foundation for computer science. Addison-Wesley Publishing Company, Amsterdam, 2nd edition, 1994.

[9] P. A. Hendriks and M. F. Singer. Solving difference equations in finite terms. J. Symbolic Comput., 27(3):239-259, 1999.

[10] M. Karr. Summation in finite terms. Journal of the ACM, 28:305-350, 1981.

[11] M. Karr. Theory of summation in finite terms. J. Symbolic Comput., 1:303-315, 1985.

[12] P. Paule and C. Schneider. Computer proofs of a new family of harmonic number identities. Adv. in Appl. Math., 31(2):359-378, 2003.

[13] P. Paule and M. Schorn. A Mathematica version of Zeilberger's algorithm for proving binomial coefficient identities. J. Symbolic Comput., 20(5-6):673-698, 1995.

[14] M. Petkovšek, H. S. Wilf, and D. Zeilberger. $A=B$. A. K. Peters, Wellesley, MA, 1996.

[15] R. Risch. The problem of integration in finite terms. Trans. Amer. Math. Soc., 139:167-189, 1969.

[16] R. Risch. The solution to the problem of integration in finite terms. Bull. Amer. Math. Soc., 76:605-608, 1970.

[17] S. Roman and G.-C. Rota. The umbral calculus. Adv. Math., 27:95-188, 1978.

[18] C. Schneider. Symbolic summation in difference fields. Technical Report 01-17, RISC-Linz, J. Kepler University, November 2001. PhD Thesis.

[19] C. Schneider. A collection of denominator bounds to solve parameterized linear difference equations in $\Pi \Sigma-$ extensions. In D. Petcu, V. Negru, D. Zaharie, and T. Jebelean, editors, Proc. SYNASC04, 6th Internat. Symposium on Symbolic and Numeric Algorithms for Scientific Computation, pages 269-282, Timisoara (Romania), September 2004. Mirton Publishing.

[20] C. Schneider. The summation package Sigma: Underlying principles and a rhombus tiling application. Discrete Math. Theor. Comput. Sci., 6(2):365-386, 2004.

[21] C. Schneider. Degree bounds to find polynomial solutions of parameterized linear difference equations in

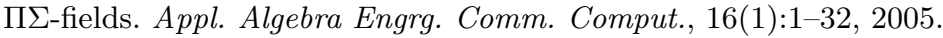

[22] C. Schneider. Solving parameterized linear difference equations in terms of indefinite nested sums and products. J. Differ. Equations Appl., 11(9):799-821, 2005.

[23] J. A. C. Weideman. http://dip.sun.ac.za/〜 weideman/PADE.

[24] J. A. C. Weideman. Padé approximations to the logarithm I: Derivation via differential equations. Quaestiones Mathematicae, 28:375-390, 2005.

[25] D. Zeilberger. A fast algorithm for proving terminating hypergeometric identities. Discrete Math., 80(2):207-211, 1990. 
(K. Driver) The John Knopfmacher Centre for Applicable Analysis and Number Theory, School of Mathematics, University of the Witwatersrand, P. O. Wits, Johannesburg 2050, South AFricA, Supported By NRF-Grant 2047226

E-mail address: kathy@maths.wits.ac.za

(H. Prodinger) The John Knopfmacher Centre for Applicable Analysis and Number Theory, School of Mathematics, University of the Witwatersrand, P. O. Wits, Johannesburg 2050, South Africa, Supported By NRF-Grant 2053748

E-mail address: hproding@sun.ac.za

(C. Schneider) Research Institute for Symbolic Computation, Johannes Kepler University, Altenberger Str. 69, A-4040 Linz, Austria, Supported by the Austrian Academy of Sciences, By the John Knopfmacher Research Centre for Applicable Analysis and Number Theory, and by The SFB-Grant F1305 AND the Grant P16613-N12 of the Austrian FWF.

E-mail address: Carsten.Schneider@risc.uni-linz.ac.at

(J.A.C. Weideman) Department of Applied Mathematics, University of Stellenbosch, Stellenbosch 7600, South Africa, Supported by NRF-grant FA2005032300018

E-mail address: weideman@dip.sun.ac.za 